\title{
Effect of enhanced Rayleigh scattering on the random fiber laser efficiency
}

\author{
Can Yao ${ }^{1,2}$, Camille Sophie Brès ${ }^{1}$, Luc Thévenaz ${ }^{2}$ \\ 1 EPFL Swiss Federal Institute of Technology, Photonic Systems Laboratory, SCI-STI-IEL, Station 11, CH-1015 Lausanne, Switzerland \\ 2 EPFL Swiss Federal Institute of Technology, Group for Fiber Optics (GFO), SCI-STI-LT, Station 11, CH-1015 Lausanne, Switzerland \\ Author e-mail address: can.yao@epfl.ch
}

\begin{abstract}
The effect of Rayleigh scattering enhancement in an optical fiber on the random fiber laser efficiency is investigated, and the effect of different corresponding fiber loss is also studied. OCIS codes: (140.0140) Lasers and laser optics; (290.0290) Scattering (C) 2018 The Author(s)
\end{abstract}

\section{Introduction}

Random fiber laser has attracted much attention for its unique properties such as cavity-free, uni-directionality, high intensity, modeless spectra and thermal stability, which has endowed it with vast potential in application in optical communication and optical sensing technologies. Other than obtaining random fiber lasing by introducing random scattering in an photonic crystal fiber with suspension fill [1] in the traditional way, or by writing a random Bragg grating in the optical fiber to form random cavities [2], Turitsyn et al. applied the natural Rayleigh scattering in the optical fiber as the feedback 'cavity', and the Raman scattering as the gain, thus explored an innovative and convenient way to obtain random fiber lasing [3]. Since then, researchers have exploited different methods to increase the efficiency and output power of the random fiber laser, by employing a mirror or FBG to form half-open cavity [4], by polarization maintaining fiber [5,6], by fiber length optimization [7], or by hybrid with active fiber [8].

Although several researchers have experimentally tracked the amplification of Rayleigh scattering feedback in an optical fiber to improve the random fiber laser efficiency $[9,10]$, there hasn't been a thorough study of the effect of Rayleigh scattering enhancement on the random fiber laser efficiency. Recently, Kashyap et al. reported that the Rayleigh scattering can be enhanced by several orders of magnitude by simple UV irradiation of the optical fiber, while little fiber loss is introduced [11,12], so it is of great interest to investigate the Rayleigh scattering and loss enhancement effect on the random fiber laser efficiency, for the optimization design of the random fiber laser by the Rayleigh scattering and loss manipulation in the optical fiber.

\section{Theory and results}

To benefit our study, we take an example scheme with pump wavelength $1090 \mathrm{~nm}$, and its corresponding $1^{\text {st }}$ and $2^{\text {nd }}$ order Stokes wavelengths are $1140 \mathrm{~nm}$ and $1210 \mathrm{~nm}$ as in reference [7]. A point reflector with reflectivity 0.6 for the $1140 \mathrm{~nm}$ wavelength is placed at the pump side of the fiber to form a half-open cavity, and the other fiber end for random fiber laser output is angle-cleaved to avoid end reflection. Assuming the pump power is as high as $7 \mathrm{~W}$, with all the gain and loss coefficients as presented in reference [7], and with the steady-state light propagation equations, we get the power evolution in the forward and backward directions along the fiber length.
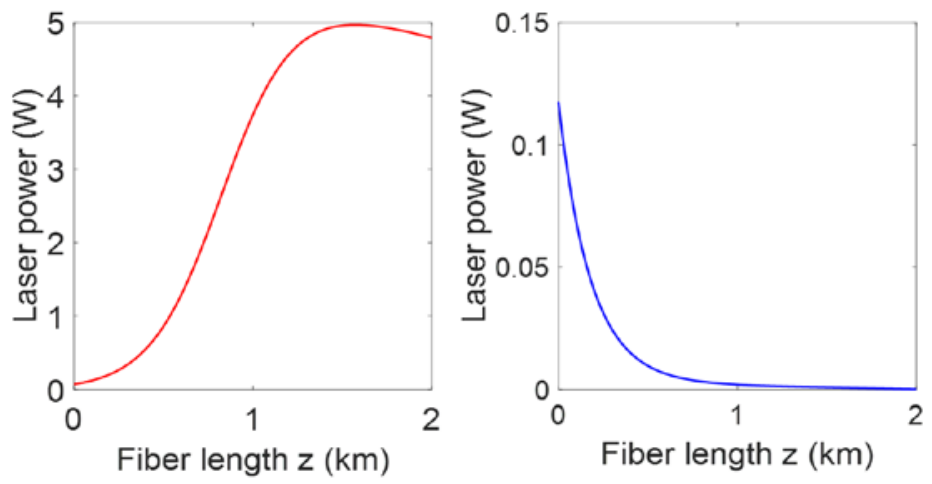

Fig. 1. The forward (red) and backward (blue) random laser power evolution along the fiber length.

In this abstract, instead of constant Rayleigh scattering coefficients $\varepsilon_{0,1,2}$ (lower indexes $0,1,2$ corresponds to the pump, $1^{\text {st }}$ and $2^{\text {nd }}$ order Stokes wavelengths), and constant fiber loss $\alpha_{0,1,2}$, we study different cases of Rayleigh 
scattering and fiber loss enhancement cases, and compare the different $1^{\text {st }}$ order random fiber laser power at the end of the fiber length at $1 \mathrm{~km}$.

1) Only Rayleigh scattering enhancement, no fiber loss enhancement. Fig. 2(a) shows that the random fiber laser in the forward direction improves no more than $3 \mathrm{~dB}$, while the random fiber laser in the backward direction can be improved by around $10 \mathrm{~dB}$, when the Rayleigh scattering is enhanced by the factor $\mathrm{n}=20 \mathrm{~dB}$ (Fig. 2(b)).
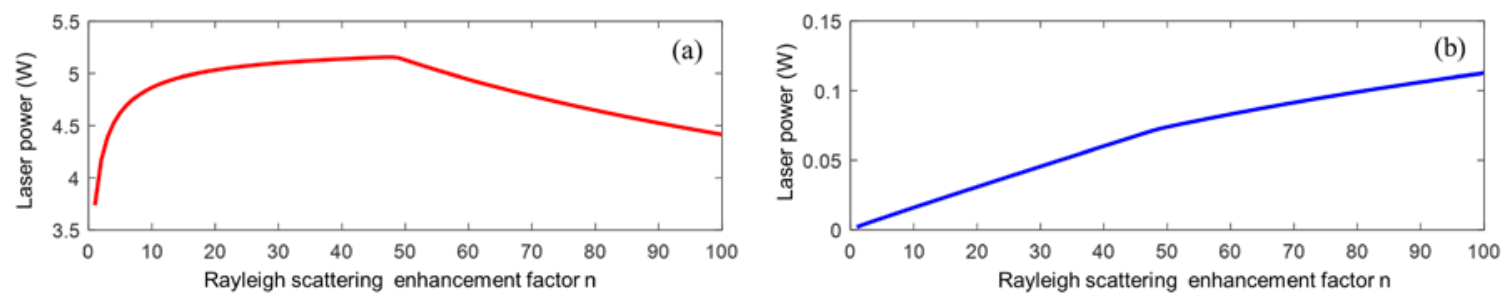

Fig. 2. The forward (a) and backward (b) laser power at $1 \mathrm{~km}$ fiber end's evolution with the Rayleigh scattering enhancement factor $\mathrm{n}$, when the loss is not enhanced.

2) Rayleigh scattering enhancement is of the same magnitude of fiber loss enhancement. From Fig. 3, we can see that the Rayleigh scattering and fiber loss enhancement is detrimental to the random laser generation both in the forward and backward directions, in spite of a small increase of the backward laser power at the beginning of the Rayleigh scattering and fiber loss enhancement.
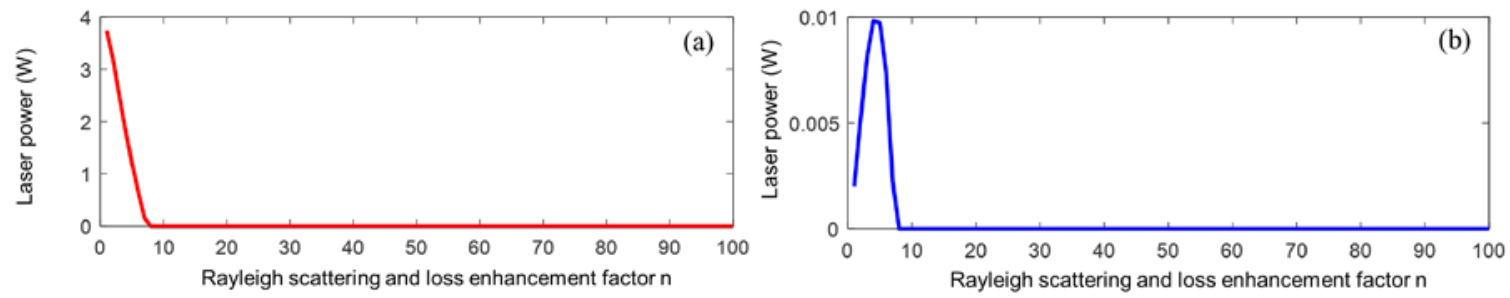

Fig. 3. The forward (a) and backward (b) laser power at $1 \mathrm{~km}$ fiber end's evolution with the Rayleigh scattering and loss enhancement factor $\mathrm{n}$.

\section{Conclusions and acknowledgement}

In summary, we conclude that Rayleigh scattering enhancement along the whole fiber length doesn't contribute much to the increase of the random fiber laser's power generation thus efficiency, and could be detrimental with the extra fiber loss introduced in the fiber. However, it is still interesting to investigate on the partial Rayleigh scattering enhancement in the fiber, for a full optimization of the random fiber laser model. The authors acknowledge the Swiss National Science Foundation (SNSF) for its financial support under number PMPDP2_164532.

\section{References}

[1] C. J. S. de Matos, L. de S. Menezes, A. M. Brito-Silva, M. a. Martinez Gámez, A. S. L. Gomes, and C. B. de Araújo, “Random Fiber Laser,” Phys. Rev. Lett., vol. 99, no. 15, p. 153903, Oct. 2007.

[2] N. Lizárraga, N. P. Puente, E. I. Chaikina, T. a Leskova, and E. R. Méndez, "Single-mode Er-doped fiber random laser with distributed Bragg grating feedback,” Opt. Express, vol. 17, no. 2, p. 395, 2009.

[3] S. K. Turitsyn, S. A. Babin, A. E. El-taher, P. Harper, D. V Churkin, V. Karalekas, E. V Podivilov, S. I. Kablukov, and J. D. Aniacastan, "Random distributed feedback fibre laser,” Nat. Photonics, vol. 4, pp. 231-235, 2010.

[4] H. Wu, Z. Wang, M. Fan, L. Zhang, W. Zhang, and Y. J. Rao, “Role of the mirror's reflectivity in forward-pumped random fiber laser,” Opt. Express, vol. 23, no. 2, p. 1421, 2015.

[5] S. A. Babin, E. A. Zlobina, S. I. Kablukov, and E. V. Podivilov, "High-order random Raman lasing in a PM fiber with ultimate efficiency and narrow bandwidth,” Sci. Rep., vol. 6, p. 22625, 2016.

[6] J. Xu, P. Zhou, J. Leng, J. Wu, and H. Zhang, "Powerful linearly-polarized high-order random fiber laser pumped by broadband amplified spontaneous emission source,” Sci. Rep., vol. 6, p. 35213, 2016.

[7] Z. Wang, H. Wu, M. Fan, L. Zhang, Y. Rao, W. Zhang, and X. Jia, "High Power Random Fiber Laser With Short Cavity Length : Theoretical and Experimental Investigations,” vol. 21, no. 1, 2015.

[8] H. Wu and Z. Wang, "Fiber Laser with Hybrid Erbium - Raman Gain,” vol. 36, no. 4, pp. 844-849, 2018.

[9] W. L. Zhang, Y. Y. Zhu, Y. J. Rao, Z. N. Wang, X. H. Jia, and H. Wu, "Random fiber laser formed by mixing dispersion compensated fiber and single mode fiber,” Opt. Express, vol. 21, no. 7, pp. 8544-8549, 2013.

[10] I. D. Vatnik, D. V Churkin, E. V Podivilov, and S. a Babin, "High-efficiency generation in a short random fiber laser,” Laser Phys. Lett., vol. 11, no. 7, p. 75101, Jul. 2014.

[11] S. Loranger, M. Gagné, V. Lambin-Iezzi, and R. Kashyap, "Rayleigh scatter based order of magnitude increase in distributed temperature and strain sensing by simple UV exposure of optical fibre,” Sci. Rep., vol. 5, p. 11177, 2015.

[12] P. S. Westbrook, K. S. Feder, R. M. Ortiz, T. Kremp, E. M. Monberg, H. Wu, D. A. Simoff, and S. Shenk, "Kilometer length , low loss enhanced back scattering fiber for distributed sensing.” 25th Optical Fiber Sensors Conference (OFS), 2017. 